\title{
Optimal Filtering Algorithm based on Covariance Information using a Sequential Fusion Approach
}

\author{
R. Caballero-Águila ${ }^{1} \mathbb{C}^{\mathrm{a}}$, A. Hermoso-Carazo ${ }^{2} \mathbb{0}^{\mathrm{b}}$ and J. Linares-Pérez ${ }^{2} \mathbb{C}^{\mathrm{c}}$ \\ ${ }^{1}$ Departamento de Estadística e I.O., Universidad de Jaén, Campus Las Lagunillas s/n, 23071 Jaén, Spain \\ ${ }^{2}$ Departamento de Estadística e I.O., Universidad de Granada, Campus Fuentenueva s/n, 18071 Granada, Spain
}

\begin{abstract}
Keywords: Sequential Fusion Filtering, Random Parameter Matrices, Cross-correlated Noises, Covariance-based Estimation, Sensor Networks.

Abstract: $\quad$ The least-squares linear filtering problem is addressed for discrete-time stochastic signals, whose evolution model is unknown and only the mean and covariance functions of the processes involved in the sensor measurement equations are available instead. The sensor measured outputs are perturbed by additive noise and different uncertainties, which are modelled in a unified way by random parameter matrices. Assuming that, at each sampling time, the noises from the different sensors are cross-correlated with each other, the sequential fusion architecture is adopted and the innovation technique is used to derive an easily implementable recursive filtering algorithm. A simulation example is included to verify the effectiveness of the proposed sequential fusion filter and analyze the influence of the sensor disturbances on the filter performance.
\end{abstract}

\section{INTRODUCTION}

Due to the progress of engineering, computer science and technology, multisensor systems are extensively used with different purposes in a large variety of fields, such as target tracking, navigation guidance or process monitoring and surveillance, among others. These applications demand the necessity of efficiently using all the information contained in the multiple sets of available data, coming from the different sensors, which must be used to estimate the signal of interest. In general, the application of suitable information fusion techniques in sensor networks provide more accurate estimations and more specific inferences than traditional single-sensor systems. As it is well known, the centralized fusion method provides optimal estimators, but suffers from heavy computational burden and low sensitivity, while the distributed fusion architecture is more robust and flexible, but provides less accurate estimators in general. The sequential fusion method, where the estimator is updated by processing the sensor data one at a time in a sequential way (instead of processing them as a whole vector), overcomes these issues, achieving

\footnotetext{
a (iD https://orcid.org/0000-0001-7659-7649

b (i) https://orcid.org/0000-0001-8120-2162

c (D) https://orcid.org/0000-0002-6853-555X
}

the same estimation accuracy but a lower computational cost than the centralized one. For this reason, the sequential fusion estimation problem in multisensor systems is currently an active research topic (Feng et al., 2018), (Lin and Sun, 2018), (Wen et al., 2013), (Yan et al., 2013).

Assuming that, apart from the additive noises, there are no uncertainties in the sensor measurements and they are sent to the processing center over perfect transmissions, there exists a rich literature about sequential estimation (see e.g., (Wen et al., 2013), (Yan et al., 2013) and references therein). Nevertheless, the presence of random disturbances (stochastic parameter perturbations, missing or fading measurements, multiplicative noise, etc.) in the sensor output measurements is usually unavoidable, due to network bandwidth limitations or communication channel inaccuracies (see (Hu et al., 2017), (Li et al., 2017), (Caballero-Águila et al., 2017), (Liu et al., 2016) and (Wang and Sun, 2017), among others). The use of measurement models with random parameter matrices provides a comprehensive framework to deal with these uncertainties and the design of estimation algorithms in this class of system models has aroused the interest of the scientific community over the last few years (see (Caballero-Águila et al., 2018), (CaballeroÁguila et al., 2019), (Hu et al., 2013), (Sun et al., 2017) and references therein). 
Furthermore, practical applications usually involve measurement noises that are correlated across sensors ((Li et al., 2017), (Sun et al., 2017), (Wang and Sun, 2017)) and, also, noise cross-correlation can arise in the process of discretization of continuoustime systems (Li, 2003), or after the transformation of systems with random delays or packet-dropouts (Zhu et al., 2013).

To the best of the authors' knowledge, most sequential fusion estimation algorithms existing in the literature require the knowledge of the signal evolution model. Hence, the use of covariance information instead is a novelty and an interesting research challenge. Motivated by the above considerations, our aim in this paper is to design a covariance-based sequential fusion algorithm for the least-squares linear estimator of a stochastic signal measured by a multisensor network, when the sensor outputs are perturbed by: 1) stochastic uncertainties modelled by independent random parameter matrices and 2) additive noises modelled by cross-correlated random sequences.

The rest of the paper is organised as follows. In Section 2, the observation model and the hypotheses about the stochastic processes involved are described. The innovation technique, which will be used to derive the estimation algorithm, is detailed in Section 3. The sequential fusion filtering algorithm is presented in Section 4, together with the formulas of the estimation error covariance matrices. In Section 5, the feasibility and effectiveness of the proposed sequential filter is verified by a computer simulation example. Finally, some concluding remarks are drawn in Section 6.

Notation: $\mathbb{R}^{n}$ denotes the $n$-dimensional Euclidean space and $\mathbb{R}^{n_{1} \times n_{2}}$ the set of all $n_{1} \times n_{2}$ real matrices. For a vector $\xi$ or a matrix $\Phi$, we denote by $\xi^{T}$ and $\Phi^{T}$ their transposes, and by $\Phi^{-1}$ to the inverse of $\Phi$. If the dimensions of a vector or a matrix are not explicitly stated, they are assumed to be compatible with algebraic operations. $I$ and 0 denote the identity matrix and the zero matrix, respectively. Finally, $\delta_{k, h}$ denotes the Kronecker delta function which is equal to one, if $k=h$, and zero otherwise.

\section{OBSERVATION MODEL}

This paper is concerned with the optimal least-squares (LS) linear estimation problem, using the sequential fusion technique, for discrete-time random signals from multisensor measurements with uncertainties described by random parameter matrices and crosscorrelated sensor additive noises.
Let us consider a second-order signal process whose evolution model is unknown and only information about its second-order statistical properties is available; namely, its mean and covariance functions are assumed to be known.

More specifically, consider a networked system with $m$ sensor nodes which, at each sampling time, $k \geq 1$, and for each $i=1, \ldots, m$, provide measured outputs, $z_{k}^{(i)} \in \mathbb{R}^{n_{z}}$, of the signal vector, $x_{k} \in \mathbb{R}^{n_{x}}$, according to the following observation model (see e.g. (Caballero-Águila et al., 2016)):

$$
z_{k}^{(i)}=H_{k}^{(i)} x_{k}+v_{k}^{(i)}, \quad k \geq 1 ; \quad i=1, \ldots, m,
$$

on which the following hypotheses are imposed:

(a) The signal process, $\left\{x_{k}\right\}_{k>1}$, has zero mean and its autocovariance function is expressed in the following separable form:

$$
E\left[x_{k} x_{h}^{T}\right]=\Lambda_{k} \Psi_{h}^{T}, h \leq k,
$$

where, for $k \geq 1, \Lambda_{k}, \Psi_{k} \in \mathbb{R}^{n_{x} \times n}$ are known matrices.

(b) $\left\{H_{k}^{(i)}\right\}_{k \geq 1}, \quad i=1, \ldots, m$, are independent sequences of independent random parameter matrices, whose entries $h_{k, r s}^{(i)}$, for $r=1, \ldots, n_{z}$ and $s=1, \ldots, n_{x}$, have known means $E\left[h_{k, r s}^{(i)}\right]$ and covariances $\operatorname{Cov}\left[h_{k, r s}^{(i)}, h_{k, p q}^{(i)}\right]$, for $r, p=1, \ldots, n_{z}$ and $s, q=1, \ldots, n_{x}$.

(c) The additive noises $\left\{v_{k}^{(i)}\right\}_{k \geq 1}, i=1, \ldots, m$, are white processes with zero mean and known second-order moments:

$$
E\left[v_{k}^{(i)} v_{h}^{(j) T}\right]=R_{k}^{(i, j)} \delta_{k, h} ; \quad i, j=1, \ldots, m .
$$

(d) For $i=1, \ldots, m$, the processes $\left\{x_{k}\right\}_{k \geq 1},\left\{v_{k}^{(i)}\right\}_{k \geq 1}$ and $\left\{H_{k}^{(i)}\right\}_{k>1}$ are mutually independent.

Remark 1. From hypotheses $(a),(b)$ and $(d)$, denoting $\bar{H}_{k}^{(i)} \equiv E\left[H_{k}^{(i)}\right]$ and $\widetilde{H}_{k}^{(i)} \equiv H_{k}^{(i)}-\bar{H}_{k}^{(i)}$, we have that $E\left[\widetilde{H}_{k}^{(i)} x_{k} x_{k}^{T} \widetilde{H}_{k}^{(i) T}\right]=E\left[\widetilde{H}_{k}^{(i)} \Lambda_{k} \Psi_{k}^{T} \widetilde{H}_{k}^{(i) T}\right], \quad i=1, \ldots, m$, where, for $r, s=1, \ldots, n_{z}$, the $(r, s)$-th entries of these matrices are given by:

$$
\left(E\left[\widetilde{H}_{k}^{(i)} \Lambda_{k} \Psi_{k}^{T} \widetilde{H}_{k}^{(i) T}\right]\right)_{r s}=\sum_{a=1}^{n_{x}} \sum_{b=1}^{n_{x}} \operatorname{Cov}\left[h_{k, r a}^{(i)}, h_{k, s b}^{(i)}\right]\left(\Lambda_{k} \Psi_{k}^{T}\right)_{a b} .
$$

\section{INNOVATION APPROACH TO THE ESTIMATION PROBLEM}

The innovation technique will be used to design a recursive algorithm for the sequential filtering estimators, $\widehat{x}_{k / k}^{(S)}$, of the signal $x_{k}$ based on the observations 
provided by all the sensors up to time $k$. In this Section, after introducing some basic notation, which will be used hereafter, an expression for the innovation process will be deduced.

\subsection{Basic Notation}

In the study of the sequential estimation problem we will use the following notation:

- For $i=1, \ldots, m, \mathbb{Z}_{k}^{(i)} \equiv\left\{z_{k}^{(1)}, \ldots, z_{k}^{(i)}\right\}$ denotes the set of measurements provided by the sensors $1,2, \ldots, i$, at time $k$.

- We will write $\mathbb{Z}_{k}^{(0)}$ to mean that no measurements are available.

- Since, for $1 \leq h \leq k, \mathbb{Z}_{h}^{(m)}$ is the set of measurements provided by all the sensors at time $h$, then $\mathbb{Z}_{k} \equiv\left\{\mathbb{Z}_{1}^{(m)}, \ldots, \mathbb{Z}_{k}^{(m)}\right\}$ is clearly the set of all the observations produced by all the sensors from the initial time instant up to time $k$.

- The LS linear estimator of a random vector $\alpha_{k}$ based on the observations $\left\{\mathbb{Z}_{k-1}, \mathbb{Z}_{k}^{(i)}\right\}$ will be denoted by $\widehat{\alpha}_{k / k, i}$.

Without loss of generality, we will suppose that, at each sampling time $k$, the measurements arrive in the fusion center according to the sensor numbering order; that is, for $1 \leq i<j \leq m$, the observation from the $i$-th sensor, $z_{k}^{(i)}$, is received before the observation from the $j$-th sensor, $z_{k}^{(j)}$.

\subsection{Innovation Process}

For each fixed time instant $k$, the aim is to obtain, $\widehat{x}_{k / k, i}$, the LS linear estimator of $x_{k}$ based on the observations $\left\{\mathbb{Z}_{k-1}, \mathbb{Z}_{k}^{(i)}\right\}$ by a recursive algorithm in $i=1, \ldots, m$, and, for this purpose, an innovation approach will be used.

For a fixed $k$, we consider the observation $z_{k}^{(i)}$ and we denote $\widehat{z}_{k / k, i-1}^{(i)}$ the estimator of $z_{k}^{(i)}$ based on $\left\{\mathbb{Z}_{k-1}, \mathbb{Z}_{k}^{(i-1)}\right\}$. Clearly, $\widehat{z}_{k / k, i-1}^{(i)}$ is the part of $z_{k}^{(i)}$ determined by the knowledge of the set of observations $\left\{\mathbb{Z}_{k-1}, \mathbb{Z}_{k}^{(i-1)}\right\}$ and, hence, the new information or the innovation provided by $z_{k}^{(i)}$ is given by the difference vector $\eta_{k / k, i-1}^{(i)} \equiv z_{k}^{(i)}-\widehat{z}_{k / k, i-1}^{(i)}$. As it is known (Kailath et al., 2000), the innovation process is a white noise with zero mean and covariances $\Xi_{k / k, i-1}^{(i)} \equiv E\left[\eta_{k / k, i-1}^{(i)} \eta_{k / k, i-1}^{(i) T}\right]$, and the LS linear estimator based on the observations agrees with that based on the innovations.
Hence, for $i=0, \ldots, m$, the LS linear estimator, $\widehat{\alpha}_{k / k, i}$, of a random vector $\alpha_{k}$ based on the observations $\left\{\mathbb{Z}_{k-1}, \mathbb{Z}_{k}^{(i)}\right\}$ can be expressed as a linear combination of the innovations; specifically, we have the following general expression:

$$
\begin{aligned}
\widehat{\alpha}_{k / k, i}= & \left(1-\delta_{k, 1}\right) \sum_{h=1}^{k-1} \sum_{j=1}^{m} E\left[\alpha_{k} \eta_{h / h, j-1}^{(j) T}\right] \Xi_{h / h, j-1}^{(j)-1} \eta_{h / h, j-1}^{(j)} \\
& +\left(1-\delta_{i, 0}\right) \sum_{j=1}^{i} E\left[\alpha_{k} \eta_{k / k, j-1}^{(j) T}\right] \Xi_{k / k, j-1}^{(j)-1} \eta_{k / k, j-1}^{(j)}, k \geq 1 .
\end{aligned}
$$

To obtain the innovation $\eta_{k / k, i-1}^{(i)}=z_{k}^{(i)}-\widehat{z}_{k / k, i-1}^{(i)}$, we start from (1) and, applying the projection theory, we have:

$\eta_{k / k, i-1}^{(i)}=z_{k}^{(i)}-\bar{H}_{k}^{(i)} \widehat{x}_{k / k, i-1}-\widehat{v}_{k / k, i-1}^{(i)}, \quad i=1, \ldots, m$.

Due to the correlation of the sensor noises, the noise estimator $\widehat{v}_{k / k, i-1}^{(i)}$ is not negligible and it can be derived from the general expression (2). Actually, taking into account that $E\left[v_{k}^{(i)} \eta_{h / h, j-1}^{(j) T}\right]=0$, for $h \leq k-1$ and $j=1, \ldots, m$, and denoting $\mathcal{V}_{k}^{(i, j)} \equiv$ $E\left[v_{k}^{(i)} \eta_{k / k, j-1}^{(j) T}\right]$, from (2), we obtain that:

$$
\widehat{v}_{k / k, i-1}^{(i)}=\left(1-\delta_{i, 1}\right) \sum_{j=1}^{i-1} \mathcal{V}_{k}^{(i, j)} \Xi_{k / k, j-1}^{(j)-1} \eta_{k / k, j-1}^{(j)} .
$$

Hence, we have that

$$
\begin{aligned}
\eta_{k / k, i-1}^{(i)}= & z_{k}^{(i)}-\bar{H}_{k}^{(i)} \widehat{x}_{k / k, i-1} \square \\
& -\left(1-\delta_{i, 1}\right) \sum_{j=1}^{i-1} \mathcal{V}_{k}^{(i, j)} \Xi_{k / k, j-1}^{(j)-1} \eta_{k / k, j-1}^{(j)} .
\end{aligned}
$$

Next, using again (2) and denoting $x_{k, h}^{(j)} \equiv$ $E\left[x_{k} \eta_{h / h, j-1}^{(j)}\right]$, the LS linear estimator $\widehat{x}_{k / k, i-1}$ is expressed as a linear combination of the innovations as follows:

$$
\begin{aligned}
\widehat{x}_{k / k, i-1}= & \left(1-\delta_{k, 1}\right) \sum_{h=1}^{k-1} \sum_{j=1}^{m} x_{k, h}^{(j)} \Xi_{h / h, j-1}^{(j)-1} \eta_{h / h, j-1}^{(j)} \\
& +\left(1-\delta_{i, 1}\right) \sum_{j=1}^{i-1} x_{k, k}^{(j)} \Xi_{k / k, j-1}^{(j)-1} \eta_{k / k, j-1}^{(j)} .
\end{aligned}
$$

Hence, for each time $k$, the innovation $\eta_{k / k, i-1}^{(i)}$, for $i=1, \ldots, m$, satisfies:

$$
\begin{aligned}
\eta_{k / k, i-1}^{(i)}= & z_{k}^{(i)}-\left(1-\delta_{k, 1}\right) \bar{H}_{k}^{(i)} \sum_{h=1}^{k-1} \sum_{j=1}^{m} x_{k, h}^{(j)} \Xi_{h / h, j-1}^{(j)-1} \eta_{h / h, j-1}^{(j)} \\
& -\left(1-\delta_{i, 1}\right) \sum_{j=1}^{i-1}\left(\bar{H}_{k}^{(i)} x_{k, k}^{(j)}+\mathcal{V}_{k}^{(i, j)}\right) \Xi_{k / k, j-1}^{(j)-1} \eta_{k / k, j-1}^{(j)} .
\end{aligned}
$$




\section{SEQUENTIAL FUSION ESTIMATION PROBLEM}

In this Section, using the sequential fusion method, a recursive algorithm is derived for the LS linear filtering estimators, $\widehat{x}_{k / k}^{(S)}, k \geq 1$, of the signal $x_{k}$ based on the observations $\mathbb{Z}_{k}$ yielded by all the sensors up to time $k$. The performance of these estimators is measured by the filtering error covariance matrices, $\Sigma_{k / k}^{(S)} \equiv E\left[\left(x_{k}-\widehat{x}_{k / k}^{(S)}\right)\left(x_{k}-\widehat{x}_{k / k}^{(S)}\right)^{T}\right], k \geq 1$, and, since their computation is not included in the algorithm, an expression for these matrices is also presented at the end of this section.

\subsection{Sequential Fusion Filtering Algorithm}

Under the hypotheses (a)-(d), for each $k \geq 1$, the estimators, $\widehat{x}_{k / k, i}$ are sequentially obtained by the following recursive algorithm:

$$
\widehat{x}_{k / k, i}=\Lambda_{k}\left(O_{k-1}+o_{k, i}\right), i=1, \ldots, m,
$$

where, starting from $o_{k, 0}=0$ and $O_{0}=0$, the vectors $o_{k, i}$ and $O_{k}$ are recursively obtained from

$$
\begin{aligned}
& o_{k, i}=o_{k, i-1}+M_{k}^{(i)} \Xi_{k / k, i-1}^{(i)-1} \eta_{k / k, i-1}^{(i)}, \quad i=1, \ldots, m, \\
& O_{k}=O_{k-1}+o_{k, m}, \quad k \geq 1 .
\end{aligned}
$$

The matrices $M_{k}^{(i)} \equiv E\left[o_{k, i} \eta_{k / k, i-1}^{(i) T}\right]$ are given by

$$
\begin{aligned}
M_{k}^{(i)}= & \Psi_{k}^{T} \bar{H}_{k}^{(i) T}-\left(K_{k-1}^{O}+K_{k, i-1}^{o}\right) \Lambda_{k}^{T} \bar{H}_{k}^{(i) T} \\
& -\left(1-\delta_{i, 1}\right) \sum_{j=1}^{i-1} M_{k}^{(j)} \Xi_{k / k, j-1}^{(j)-1} \mathcal{V}_{k}^{(i, j) T}, i=1, \ldots, m .
\end{aligned}
$$

The matrices $K_{k, i}^{o} \equiv E\left[o_{k, i} o_{k, i}^{T}\right]$ and $K_{k}^{O} \equiv E\left[O_{k} O_{k}^{T}\right]$ are obtained from

$$
\begin{aligned}
& K_{k, i}^{o}=K_{k, i-1}^{o}+M_{k}^{(i)} \Xi_{k / k, i-1}^{(i)-1} M_{k}^{(i) T}, i=1, \ldots, m, \\
& K_{k}^{O}=K_{k-1}^{O}+K_{k, m}^{o}, \quad k \geq 1,
\end{aligned}
$$

with initial conditions $K_{k, 0}^{o}=0$ and $K_{0}^{O}=0$.

For $i=1, \ldots, m$, the innovation $\eta_{k / k, i-1}^{(i)}$ is calculated by

$$
\begin{aligned}
\eta_{k / k, i-1}^{(i)}= & z_{k}^{(i)}-\bar{H}_{k}^{(i)} \Lambda_{k}\left(O_{k-1}+o_{k, i-1}\right) \\
& -\left(1-\delta_{i, 1}\right) \sum_{j=1}^{i-1} \mathcal{V}_{k}^{(i, j)} \Xi_{k / k, j-1}^{(j)-1} \eta_{k / k, j-1}^{(j)}
\end{aligned}
$$

and the innovation covariance matrix, $\Xi_{k / k, i-1}^{(i)}$, is given by

$$
\Xi_{k / k, i-1}^{(i)}=\bar{H}_{k}^{(i)} \Lambda_{k} M_{k}^{(i)}+\mathcal{V}_{k}^{(i, i)}, i=1, \ldots, m .
$$

The matrices $\mathcal{V}_{k}^{(i, j)}=E\left[v_{k}^{(i)} \eta_{k / k, j-1}^{(j) T}\right]$, for $i=1, \ldots, m$ and $j=1, \ldots, i$, satisfy

$$
\begin{aligned}
\mathcal{V}_{k}^{(i, j)}=R_{k}^{(i, j)}-(1 & \left.-\delta_{j, 1}\right) \sum_{l=1}^{j-1} \mathcal{V}_{k}^{(i, l)} \Xi_{k / k, l-1}^{(l)-1} \\
& \times\left(\bar{H}_{k}^{(j)} \Lambda_{k} M_{k}^{(l)}+\mathcal{V}_{k}^{(j, l)}\right)^{T} .
\end{aligned}
$$

Finally, the sequential fusion filtering estimators are given by

$$
\widehat{x}_{k / k}^{(S)}=\widehat{x}_{k / k, m}, \quad k \geq 1
$$

\subsubsection{Algorithm Derivation}

From (2), to obtain the LS linear estimators $\widehat{x}_{k / k, i}$, we start by calculating the coefficients $x_{k, h}^{(j)}=$ $E\left[x_{k} \eta_{h / h, j-1}^{(j)}\right]$, for $h \leq k$ and $j=1, \ldots, m$.

From hypotheses $(a),(b)$ and $(d)$, we have that

$$
E\left[x_{k} z_{h}^{(j) T}\right]=\Lambda_{k} \Psi_{h}^{T} \bar{H}_{h}^{(j) T}, h \leq k, j=1, \ldots, m .
$$

Using this property together with expression (4) for $\eta_{h / h, j-1}^{(j)}$, we have that, for $h \leq k$ and $j=1, \ldots, m$ :

$$
\chi_{k, h}^{(j)}=\Lambda_{k} \Psi_{h}^{T} \bar{H}_{h}^{(j) T}
$$

$$
\begin{aligned}
& -\left(1-\delta_{h, 1}\right) \sum_{l=1}^{h-1} \sum_{s=1}^{m} X_{k, l}^{(s)} \Xi_{l / l, s-1}^{(s)-1} X_{h, l}^{(s) T} \bar{H}_{h}^{(j) T} \\
& -\left(1-\delta_{j, 1}\right) \sum_{s=1}^{j-1} X_{k, h}^{(s)} \Xi_{h / h, s-1}^{(s)-1}\left(\bar{H}_{h}^{(j)} \chi_{h, h}^{(s)}+\mathcal{V}_{h}^{(j, s)}\right)^{T} .
\end{aligned}
$$

Hence, $X_{k, h}^{(j)}$ can be expressed as

$$
x_{k, h}^{(j)}=\Lambda_{k} M_{h}^{(j)}, h \leq k ; j=1, \ldots, m,
$$

where $M_{h}^{(j)}$ is a function satisfying

$$
\begin{aligned}
M_{h}^{(j)}= & \Psi_{h}^{T} \bar{H}_{h}^{(j) T} \\
& -\left(1-\delta_{h, 1}\right) \sum_{l=1}^{h-1} \sum_{s=1}^{m} M_{l}^{(s)} \Xi_{l / l, s-1}^{(s)-1} M_{l}^{(s) T} \Lambda_{h}^{T} \bar{H}_{h}^{(j) T} \\
& -\left(1-\delta_{j, 1}\right) \sum_{s=1}^{j-1} M_{h}^{(s)} \Xi_{h / h, s-1}^{(s)-1}\left(\bar{H}_{h}^{(j)} \Lambda_{h} M_{h}^{(s)}+\mathcal{V}_{h}^{(j, s)}\right)^{T} .
\end{aligned}
$$

Now, taking into account that, from (2),

$$
\begin{aligned}
\widehat{x}_{k / k, i}=(1 & \left.-\delta_{k, 1}\right) \sum_{h=1}^{k-1} \sum_{j=1}^{m} x_{k, h}^{(j)} \Xi_{h / h, j-1}^{(j)-1} \eta_{h / h, j-1}^{(j)} \\
& +\left(1-\delta_{i, 0}\right) \sum_{j=1}^{i} x_{k, k}^{(j)} \Xi_{k / k, j-1}^{(j)-1} \eta_{k / k, j-1}^{(j)},
\end{aligned}
$$

using expression (13) and defining the vectors

$$
\begin{aligned}
o_{k, i} & \equiv \sum_{j=1}^{i} M_{k}^{(j)} \Xi_{k / k, j-1}^{(j)-1} \eta_{k / k, j-1}^{(j)}, \quad i=1, \ldots, m, \\
O_{k} & \equiv \sum_{h=1}^{k} o_{h, m}, \quad k \geq 1
\end{aligned}
$$


which, obviously, satisfy expression (6), we conclude that the estimator $\widehat{x}_{k / k, i}$ is given by (5).

Now, from (14) for $h=k$ and $j=i$, defining the matrices

$$
\begin{gathered}
K_{k, i}^{o} \equiv E\left[o_{k, i} o_{k, i}^{T}\right]=\sum_{j=1}^{i} M_{k}^{(j)} \Xi_{k / k, j-1}^{(j)-1} M_{k}^{(j) T}, \\
K_{k}^{O} \equiv E\left[O_{k} O_{k}^{T}\right]=\sum_{h=1}^{k} K_{h, m}^{o},
\end{gathered}
$$

expression (7) is obtained.

The recursive formula (8) for the matrices $K_{k, i}^{o}$ and $K_{k}^{O}$ is obvious from (15).

Expression (9) for the innovation $\eta_{k / k, i-1}^{(i)}$ is easily obtained by substituting (5) for $\widehat{x}_{k / k, i-1}^{(i)}$ in (3).

To deduce expression (10) for the covariance matrix $\Xi_{k / k, i-1}^{(i)}=E\left[\eta_{k / k, i-1}^{(i)} \eta_{k / k, i-1}^{(i) T}\right]$, we express $\Xi_{k / k, i-1}^{(i)}=E\left[z_{k}^{(i)} \eta_{k / k, i-1}^{(i) T}\right]$, and use expression (1) for $z_{k}^{(i)}$. Then, since $E\left[x_{k} \eta_{k / k, i-1}^{(i) T}\right]=X_{k, k}^{(i)}=\Lambda_{k} M_{k}^{(i)}$, and $\left.E\left[v_{k}^{(i)}\right) \eta_{k / k, i-1}^{(i) T}\right]=\mathcal{V}_{k}^{(i, i)}$, expression (10) is straightforward.

To obtain (11) for $\mathcal{V}_{k}^{(i, j)}=E\left[v_{k}^{(i)} \eta_{k / k, j-1}^{(j) T}\right]$ we use (4) for $\eta_{k / k, j-1}^{(j)}$; so, taking into account that $E\left[v_{k}^{(i)} z_{k}^{(j) T}\right]=R_{k}^{(i, j)}, E\left[v_{k}^{(i)} \eta_{h / h, l-1}^{(l) T}\right]=0$, for $h \leq k-1$, and $E\left[v_{k}^{(i)} \eta_{k / k, l-1}^{(l) T}\right]=\mathcal{V}_{k}^{(i, l)}$, expression (11) is obtained.

Finally, since $\mathbb{Z}_{k}=\left\{\mathbb{Z}_{k-1}, \mathbb{Z}_{k}^{(m)}\right\}$, it is obvious that the sequential fusion filter is given by (12), and the algorithm is proven.

\subsection{Sequential Filtering Error Covariance Matrices}

Under the hypotheses (a)-(d), for each time instant $k \geq 1$, the estimation error covariance matrices, $\Sigma_{k / k, i} \equiv E\left[\left(x_{k}-\widehat{x}_{k / k, i}\right)\left(x_{k}-\widehat{x}_{k / k, i}\right)^{T}\right]$, are sequentially obtained by the following formula:

$$
\Sigma_{k / k, i}=\Lambda_{k}\left(\Psi_{k}-\Lambda_{k}\left(K_{k-1}^{O}+K_{k, i}^{o}\right)\right)^{T}, i=1, \ldots, m,
$$

where the matrices $K_{k}^{O}$ and $K_{k, i}^{o}$ are calculated by (8). The sequential fusion filtering error covariance matrix, $\Sigma_{k / k}^{(S)}=E\left[\left(x_{k}-\widehat{x}_{k / k}^{(S)}\right)\left(x_{k}-\widehat{x}_{k / k}^{(S)}\right)^{T}\right]$, is given by

$$
\Sigma_{k / k}^{(S)}=\Sigma_{k / k, m}, \quad k \geq 1 .
$$

Proof. From hypothesis $(a), E\left[x_{k} x_{k}^{T}\right]=\Lambda_{k} \Psi_{k}^{T}$; then, expression for $\Sigma_{k / k, i}$ is clear taking into account that

$$
\Sigma_{k / k, i}=E\left[x_{k} x_{k}^{T}\right]-E\left[\widehat{x}_{k / k, i} \widehat{x}_{k / k, i}^{T}\right],
$$

and using (5) for the estimators $\widehat{x}_{k / k, i}$.

From (12) for $\widehat{x}_{k / k}^{(S)}$, is immediate that the sequential error covariance matrices are $\Sigma_{k / k}^{(S)}=\Sigma_{k / k, m}$.

Note that the matrices $\Sigma_{k / k, i}$ only depend on the matrices $\Lambda_{k}$ and $\Psi_{k}$, which are known according to hypothesis $(a)$, and on the matrices $K_{k}^{O}$ and $K_{k, i}^{o}$, which are recursively calculated by (8). Thus, $\Sigma_{k / k, i}$ do not depend on the observations and, hence, these matrices provide a measure of the performance of the estimators $\widehat{x}_{k / k, i}, i=1, \ldots, m$, (and, in particular, of $\left.\widehat{x}_{k / k}^{(S)}\right)$ even before we get any measurement data.

\section{SIMULATION EXAMPLE}

This section is devoted to analyze the effectiveness of the proposed sequential fusion filtering algorithm by a numerical simulation example. On the one hand, this example illustrates how hypothesis $(a)$ can cover situations with state-dependent multiplicative noise and, on the other, it shows how the proposed model with random parameter matrices can describe different kinds of sensor uncertainties.

Consider the same signal process as that in Example 1 of (Feng et al., 2013); that is, a two-dimensional signal $x_{k}$ generated by the following model with statedependent multiplicative noise:

$$
x_{k+1}=\left(F+\varepsilon_{k} G\right) x_{k}+\rho w_{k}, \quad k \geq 1,
$$

where

$$
F=\left(\begin{array}{cc}
0.95 & 0.01 \\
0 & 0.95
\end{array}\right), G=\left(\begin{array}{cc}
0.01 & 0 \\
0 & 0.01
\end{array}\right), \rho=\left(\begin{array}{l}
0.8 \\
0.6
\end{array}\right) \text {. }
$$

The noise sequences $\left\{\varepsilon_{k}\right\}_{k \geq 1}$ and $\left\{w_{k}\right\}_{k \geq 1}$ are standard white gaussian scalar noises. The initial signal $x_{0}$ is a gaussian two-dimensional random vector with zero mean and covariance matrix $E\left[x_{0} x_{0}^{T}\right]=I$. These noise sequences and initial signal vector are assumed to be mutually independent; then, it is easy to see that the signal covariance function is given by $E\left[x_{k} x_{h}^{T}\right]=F^{k-h} E\left[x_{h} x_{h}^{T}\right], h \leq k$, and hypothesis $(a)$ is satisfied taking $\Lambda_{k}=F^{k}$ and $\Psi_{h}^{T}=F^{-h_{\mathcal{E}_{h}}}$, where $\mathcal{E}_{h} \equiv E\left[x_{h} x_{h}^{T}\right], h \geq 1$, is recursively obtained by:

$$
\mathcal{E}_{h}=F \mathcal{E}_{h-1} F^{T}+G \mathcal{E}_{h-1} G^{T}+\rho \rho^{T}, h \geq 1 ; \quad \mathcal{E}_{0}=I .
$$

Consider scalar measurements of this signal, coming from three sensors, according to model (1):

$$
z_{k}^{(i)}=H_{k}^{(i)} x_{k}+v_{k}^{(i)}, \quad k \geq 1, \quad i=1,2,3,
$$

where the random parameter matrices $\left\{H_{k}^{(i)}\right\}_{k \geq 1}, i=$ $1,2,3$ are defined by: 
- $H_{k}^{(1)}=\mu_{k}^{(1)}(0.74,0.75)$, where $\left\{\mu_{k}^{(1)}\right\}_{k \geq 1}$ is a sequence of independent and identically distributed (iid) random variables with uniform distribution over $[0.3,0.7]$. Hence, continuous gain degradation in sensor 1 is modelled by $\left\{H_{k}^{(1)}\right\}_{k \geq 1}$.

- $H_{k}^{(2)}=\mu_{k}^{(2)}(0.75,0.70)$, where $\left\{\mu_{k}^{(2)}\right\}_{k \geq 1}$ is a sequence of iid discrete random variables with

$$
P\left[\mu_{k}^{(2)}=0.9\right]=0.8, P\left[\mu_{k}^{(2)}=0.1\right]=0.2 .
$$

Hence, the sequence $\left\{H_{k}^{(2)}\right\}_{k \geq 1}$ models discrete gain degradation in sensor 2 .

- $H_{k}^{(3)}=\mu_{k}^{(3)}(0.80,0.75)$, where $\left\{\mu_{k}^{(3)}\right\}_{k \geq 1}$ are iid Bernoulli random variables with $P\left[\mu_{k}^{(3)}=1\right]=p$, $\forall k \geq 1$. The missing measurement phenomena in sensor 3 is, hence, described by $\left\{H_{k}^{(3)}\right\}_{k \geq 1}$. Note that $1-p$ is the missing probability, which means that the signal $x_{k}$ is missing from the $k$ th measurement coming from the third sensor (or, equivalently, that this measurement is only noise: $z_{k}^{(3)}=v_{k}^{(3)}$ ) with probability $1-p$.

As in (Caballero-Águila et al., 2016), the noises $\left\{v_{k}^{(i)}\right\}_{k \geq 1}, i=1,2,3$, are defined by $v_{k}^{(i)}=c_{i} \beta_{k}, i=$ $1,2,3$, with $c_{1}=0.25, c_{2}=0.5$ and $c_{3}=0.75$, and $\left\{\beta_{k}\right\}_{k \geq 1}$ a standard Gaussian white process. Clearly, according to hypothesis $(b)$, these noises are correlated only at the same time instant and $R_{k}^{(i, j)}=c_{i} c_{j}$.

The proposed sequential fusion filtering algorithm, as well as the formulas for the error covariance matrices, have been implemented in a MATLAB program and one hundred iterations have been run to show the algorithm effectiveness. In order to illustrate the influence of the missing probability on the filter accuracy, the error variances have been calculated for different probabilities $p$ of the Bernoulli random variables modelling the missing measurement phenomena of sensor 3 .

First, considering $p=0.5$, the sequential fusion estimates have been calculated using simulated values of the signal and the observations coming from the three sensors. The results of a simulated signal together with the sequential filtering estimates are plotted in Figure 1 which, for both the first and the second signal components, shows a satisfactory tracking performance of the proposed estimates.

As indicated above, the estimation error covariance matrices, which do not depend on the simulated data, measure the accuracy of the estimators. For both signal components, in order to show the effect of the missing measurement phenomenon on the performance of the sequential filtering estimators, their error variances have been calculated for values of $p$
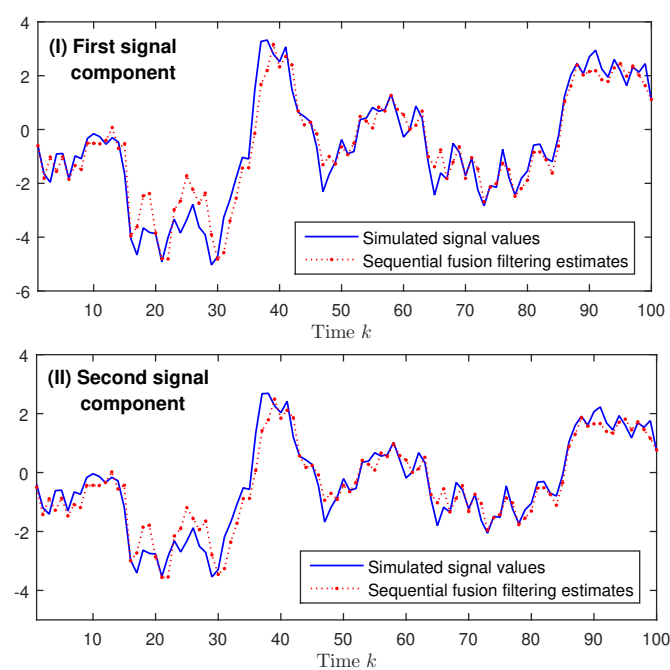

Figure 1: Simulated signal values and sequential fusion filtering estimates when $p=0.5$.

varying from 0.4 to 0.9 ; the results are displayed in Figure 2. From this figure, it is observed that the performance of the sequential filter is indeed influenced by the probabilities $p$; actually, for both signal components, the sequential filtering error variances become smaller as $1-p$ (the probability of missing measurements) decreases, which, as expected, confirms that the performance of the sequential filters improves as the probability $p$ increases. For the values $p=0.1,0.2$ and 0.3 , no appreciable differences are visually observed in comparison with the results obtained for $p=0.4$ and, therefore, these values have been omitted in Figure 2.

Finally, we study the performance of the proposed sequential fusion filtering estimators compared with the local filters and the centralized fusion filter in (Caballero-Águila et al., 2016), for which, considering $p=0.5$, the error variances of the different filters have been calculated. These filtering error variances are displayed in Figure 3, which shows that the sequential filtering error variances are smaller than those of the every local filter and, hence, the sequential fusion filtering estimators outperform all the local ones. Also, it can be observed that the error variances of the sequential filter are identical to those of the centralized filter. This result was expected, since the centralized and sequential estimators are optimal based on the same number of observations, and therefore, they are actually equal to each other. Nevertheless, the sequential fusion algorithm can significantly reduce the computational cost of the centralized fusion algorithm; indeed, in this example the computational complexity of the centralized algorithm has the order of magnitude $O\left(3^{3}\right)$ and that of the sequential algorithm has the order $O(3)$. 

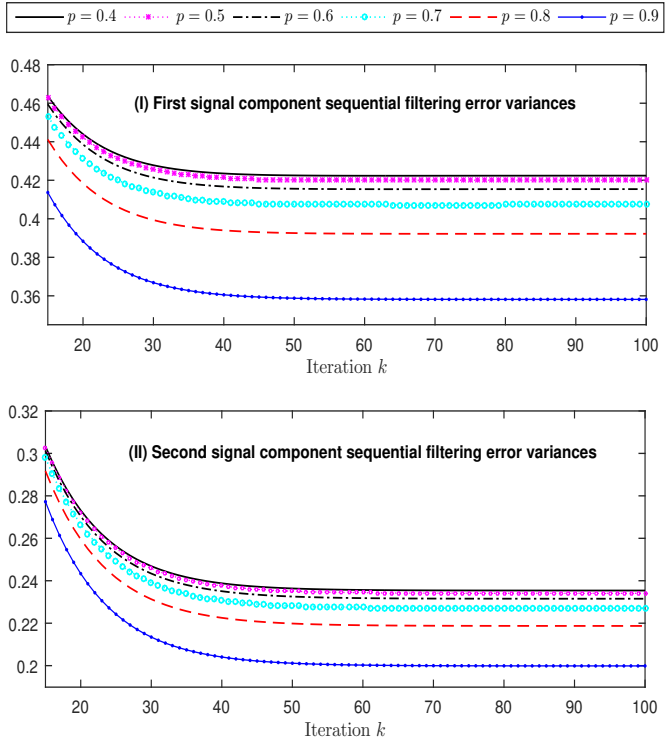

Figure 2: Sequential filtering error variances for $p$ from 0.4 to 0.9 .

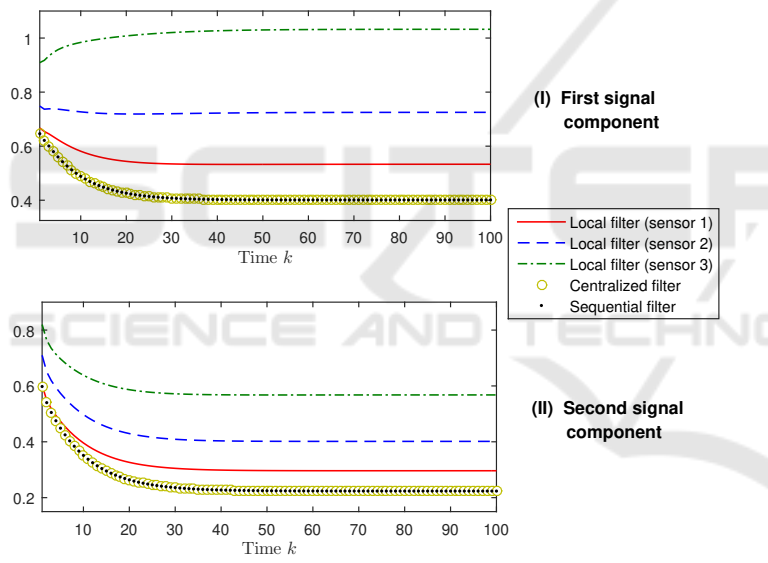

Figure 3: Error variance comparison of the local filter and the centralized and sequential fusion filtering estimators when $p=0.5$.

\section{CONCLUSIONS}

As it is known, centralized and distributed fusion estimation algorithms present some handicaps, which can be overcome by using the sequential fusion method, whose estimation accuracy is identical to the centralized one, with the difference that the sensor data are processed in a sequential way, thus having a considerably lower computational cost. A review of the sequential fusion estimation algorithms in the literature brings to light that they all require the knowledge of the signal evolution model. However, in many practical applications, such information is not available and only the first and second-order moments of the signal and the processes involved in the sensor measurement equations can be obtained. For the first time, this paper addresses the sequential fusion estimation problem using covariance information when the sensor measurement model includes random parameter matrices and additive noises that are correlated across sensors.

A simulation example shows how systems with state-dependent multiplicative noise fit the proposed covariance-based approach and how multiple uncertainties in the measurement equations (namely, discrete or continuous gain degradation and missing measurements) are covered by the current measurement model with random parameter matrices. The simulation results illustrate the applicability of the proposed sequential fusion filter and the effect of the missing measurement phenomenon on the estimation accuracy.

\section{ACKNOWLEDGEMENTS}

This research is supported by Ministerio de Economía, Industria y Competitividad, Agencia Estatal de Investigación and Fondo Europeo de Desarrollo Regional FEDER (grant no. MTM2017-84199-P).

\section{REFERENCES}

Caballero-Águila, R., Hermoso-Carazo, A., and LinaresPérez, J. (2016). Fusion estimation using measured outputs with random parameter matrices subject to random delays and packet dropouts. Signal Processing, 127:12-23.

Caballero-Águila, R., Hermoso-Carazo, A., and LinaresPérez, J. (2017). Fusion estimation from multisensor observations with multiplicative noises and correlated random delays in transmission. Mathematics, 5(3):45.

Caballero-Águila, R., Hermoso-Carazo, A., and LinaresPérez, J. (2018). Centralized fusion approach to the estimation problem with multi-packet processing under uncertainty in outputs and transmissions. Sensors, 18(8):2697.

Caballero-Águila, R., Hermoso-Carazo, A., and LinaresPérez, J. (2019). Networked distributed fusion estimation under uncertain outputs with random transmission delays, packet losses and multi-packet processing. Signal Processing, 156:71-83.

Feng, J., Wang, Z., and Zeng, M. (2013). Distributed weighted robust Kalman filter fusion for uncertain systems with autocorrelated and cross-correlated noises. Information Fusion, 14:78-86.

Feng, X., Wu, L., and Wen, C. (2018). Sequential fault-tolerant fusion estimation for multisensor time- 
varying systems. Discrete Dynamics in Nature and Society, ID 4504206:9 pages.

Hu, J., Wang, Z., Alsaadi, F. E., and Hayat, T. (2017). Event-based filtering for time-varying nonlinear systems subject to multiple missing measurements with uncertain missing probabilities. Information Fusion, 38:74-83.

Hu, J., Wang, Z., and Gao, H. (2013). Recursive filtering with random parameter matrices, multiple fading measurements and correlated noises. Automatica, 49:3440-3448.

Kailath, T., Sayed, A. H., and Hassibi, B. (2000). Linear estimation. Prentice Hall, Upper Saddle River, New Jersey.

Li, W., Jia, Y., and Du, J. (2017). Distributed filtering for discrete-time linear systems with fading measurements and time-correlated noise. Digital Signal Processing, 60:211-219.

Li, X. (2003). Optimal linear estimation fusion, part vii: Dynamic systems. In Proceedings of the 6th International Conference of Information Fusion. IEEE.

Lin, H. and Sun, S. (2018). Optimal sequential fusion estimation with stochastic parameter perturbations, fading measurements, and correlated noises. IEEE Transactions on Signal Processing, 66(13):3571-3583.

Liu, Y., Wang, Z., He, X., and Zhou, D. H. (2016). Minimum-variance recursive filtering over sensor networks with stochastic sensor gain degradation: Algorithms and performance analysis. IEEE Transactions on Control of Network Systems, 3(3):265-274.

Sun, S., Tian, T., and Lin, H. (2017). State estimators for systems with random parameter matrices, stochastic nonlinearities, fading measurements and correlated noises. Information Sciences, 397-398:118-136.

Wang, X. and Sun, S. (2017). Optimal recursive estimation for networked descriptor systems with packet dropouts, multiplicative noises and correlated noises. Aerospace Science and Technology, 63:41-53.

Wen, C., Cai, Y., Wen, C., and Xu, X. (2013). Optimal sequential Kalman filtering with cross-correlated measurement noises. Aerospace Science and Technology, 26:153-159.

Yan, L., Li, X. R., Xia, Y., and Fu, M. (2013). Optimal sequential and distributed fusion for state estimation in cross-correlated noise. Automatica, 49:3607-3612.

Zhu, C., Xia, Y., Xie, L., and Yan, L. (2013). Optimal linear estimation for systems with transmission delays and packet dropouts. IET Signal Processing, 7(9):814823. 